\title{
Robust and Adaptive Block Tracking Method based on particle filter
}

\author{
Bin Sun \\ School of Information Science \\ and Engineering \\ Shandong University \\ sunbinsdu@mail.sdu.edu.cn
}

\author{
Zhi Liu \\ School of Information Science \\ and Engineering \\ Shandong University \\ liuzhi@sdu.edu.cn
}

\author{
Haixia Zhang \\ School of Information Science \\ and Engineering \\ Shandong University \\ haixia.zhang@sdu.edu.cn
}

\begin{abstract}
In the field of video analysis and processing, object tracking is attracting more and more attention especially in traffic management, digital surveillance and so on. However problems such as objects' abrupt motion, occlusion and complex target structures would bring difficulties to academic study and engineering application. In this paper, a fragmentsbased tracking method using the block relationship coefficient is proposed. In this method, we use particle filter algorithm and object region is divided into blocks initially. The contribution of this method is that object features are not extracted just from a single block, the relationship between current block and its neighbor blocks are extracted to describe the variation of the block. Each block is weighted according to the block relationship coefficient when the block is voted on the most matched region in next frame. This method can make full use of the relationship between blocks. The experimental results demonstrate that our method can provide good performance in condition of occlusion and abrupt posture variation.
\end{abstract}

\section{Keywords}

object tracking, blocking, particle filter

\section{INTRODUCTION}

Object tracking has become the research hotspot in the field of computer vision, and is widely used in traffic management, digital surveillance, intelligent city and so on. However, problems such as occlusion, illumination variation and complex background would influence the performance of object tracking [1]. In order to get a high tracking accuracy, researchers have been putting forward lots of algorithms [2] [3] to overcome illumination variation and occlusions in the surveillance video. Traditional methods based on target pattern-matching search [4] regard targeted object tracking as a local pattern matching optimization problem. As the classic method of target pattern-matching tracking algorithm, Mean shift algorithm [5] is suitable for real-time tracking on account of its convergence. But the weak response to the size change of target limits the development of this method. Another traditional method based on filter theory transmits the targeted object tracking into the Bayesian theory framework [6]. It uses prior probability which is known previously to forecast the maximum posterior probability of the targeted object. Kalman filter algorithm [7] has a good performance dealing with the linear, Gaussian and single-model tracking task, while particle filter algorithm [8] [9] is suitable for nonlinear and non-Gaussian tracking. Particle filter has no special requirements for system model and it can keep the multimodal distribution of state. So particle filter is widely used in tracking field.

As is known to all, the accuracy of tracking can be greatly influenced by occlusion and the posture variation of the object. In order to reduce the bad impact of occlusion and posture variation in tracking process, experts proposed plenty of efficient methods. For example, Yang C proposed object tracking method based on a new metric [10] which measures the similarity of the target region and candidate region. This metric instead of the Bhattacharyya coefficient, with the color density and space information of the target region considered. Later Adam. A [11] divided the targeted object into several blocks with the same size, then calculated the color histogram of each block and searched for the most matched block in the candidate region of next frame. After the most matched blocks are found, they are weighted and voted on the possible position of the object in next frame. This method improves the accuracy of tracking in condition of occlusion. However, it costs big computations and it doesn't make full use of the relationship between each blocks.

In this paper, we propose a new fragments-based tracking algorithm. The object region is divided into several blocks with the same size, and then color histogram of each block is extracted as the feature. After that we calculate the Bhattacharyya coefficient of color histogram between current and its neighbor blocks. Candidate region of next frame is also divided into blocks and the Bhattacharyya coefficient of each block and its neighbor blocks are calculated. Then compare the variation of Bhattacharyya coefficient of block between object region and candidate region, and set the weight of each block according to the variation. This method can make full use of the relationship between blocks, and it can have a good performance in condition of occlusion and abrupt posture variation. Experimental results show this 
method is robust and provides high accuracy.

\section{FRAGMENT-BASED TRACKING ALGO- RITHM USING PARTICLE FILTER}

\subsection{Particle filter}

Particle filter is mainly used to track the motion target in condition of nonlinear and non-Gaussian, on account of its robustness against with the complex background. Particle filter uses several discrete particles to represent the main distributions of target location nearby. In the framework of Bayesian filter, as observed characters $Z^{t}=\left\{Z_{1}, Z_{2}, \ldots, Z_{t}\right\}$ are known, then the posterior distribution can be updated recursively by current state. Formula (1) is shown as below.

$$
\begin{aligned}
p\left(X_{t} \mid Z^{t}\right) & =k p\left(Z_{t} \mid X_{t}\right) p\left(X_{t} \mid Z^{t-1}\right) \\
& =k p\left(Z_{t} \mid X_{t}\right) \int\left(p\left(X_{t} \mid X_{t-1}\right) p\left(X_{t-1} \mid Z^{t-1}\right)\right)
\end{aligned}
$$

where, $p\left(Z_{t} \mid X_{t}\right)$ represents the measurement model with motion model expressed by $p\left(X_{t} \mid X_{t-1}\right)$. In the process of particle filter, posterior probability $p\left(X_{t} \mid Z^{t-1}\right)$ can approximately become the collection of weighted sample $\left\{X_{t-1}^{(i)}, w_{t-1}^{(i)}\right\}_{i=1}^{N}$ by recursively computation. Shown as

$$
p\left(X_{t} \mid Z^{t}\right) \approx k p\left(Z_{t} \mid X_{t}\right) \sum_{i=1}^{N} w_{t-1}^{(i)} p\left(X_{t} \mid X_{t-1}^{(i)}\right)
$$

The procedure of particle filter algorithm is shown as below [12].

Step1. Initialization. Extract the particle collection for prior probability $p\left(X_{0}\right)$ to generate the $\left\{X_{0}^{(i)}, w_{0}^{(i)}\right\}_{i=1}^{N}$, with time $\mathrm{k}$ equals to 1 .

Step2. Sampling. For $\mathrm{i}=1,2, \ldots, \mathrm{N}$, we sample $X_{k}^{(i)}$ from $p\left(X_{k}^{(i)}\right.$ $\left.X_{k-1}^{(i)}\right)$ to estimate the new weight value $w_{k}^{(i)}=p\left(Z_{k} \mid X_{k}^{(i)}\right)$ , then normalize the weight value according to the formula (3) below.

$$
w_{k}^{(i)}=\frac{w_{k}^{(i)}}{\sum_{j=1}^{N} w_{k}^{(i)}}, i=1,2, \ldots, N
$$

Step3. Output. Collection of particle $\left\{X_{k}^{(i)}, w_{k}^{(i)}\right\}_{i=1}^{N}$ which can be used to estimate the posterior probability $p\left(X_{k} \mid Z^{k}\right)$ is exported in this step.

Step4. Resampling. Use probability $w_{t}^{(i)}$ to resample the particle $X_{k}^{(i)}$ to get the independent and identically distributed random particle $X_{k}^{(i)}$ which can approximately obey the distribution of $p\left(X_{t} \mid Z^{k}\right)$.

Step5. $\mathrm{k}=\mathrm{k}+1$, switch to step 2 .

\subsection{Block relationship matched tracking al- gorithm}

In the field of object tracking, the region of targeted object is normally expressed by a rectangle or a ellipse. After the region of targeted object is defined in the first frame, the feature of this region is extracted and used as the metric for searching the most matched region in the following frames. However, the targeted object region may contain lots of background information which reduces the accuracy of extracted feature for describing the object. This leads to the error in searching the most matched region, so the idea of blocking [13] can solve this problem by reduce the weight of background blocks while blocks are voted for the most matched region.

In order to increase the adaption of our method, the targeted object region is divided into several $8 \times 8,16 \times 16$ and $32 \times 32$ blocks according to the region size. If the object region size is big, then we divide it into $32 \times 32$ blocks. On the contrary, the region would be divided into $8 \times 8$ blocks while its size is small.

In traditional fragments-based tracking algorithms, the feature of blocks in the candidate region in next frame is extracted and matched with the feature extracted from target region. If the feature can be well matched, the weight of this block would be increased while blocks are voted on the possible position of targeted object in next frame. The disadvantage of traditional fragments-based tracking algorithm is that they only extract the feature from one block with the relationship between neighbor blocks ignored. As we know, the feature information is got from large numbers of pixels not a single pixel. Similarly, in the process of fragmentsbased tracking method, the feature of single block cannot provide enough information. Relationship between neighbor blocks should be considered for a good description of the target region.

\section{THE PROPOSED METHOD FOR OBJECT TRACKING}

\section{1 block relationship coefficient}

In this paper, we propose a new coefficient called block relationship coefficient (BRC) which represents the variation around the current block. If the variation of block relationship coefficient between current block and candidate block in next frame is big, that means occlusion and interference is strong in or around this block. Then the weight of this block is decreased while it is voted on the most matched region in next frame. On the contrary, the weight of block would be increased if the variation of its block relationship coefficient is small. The proposed coefficient can help tracking method to reduce the influence of occlusion or posture variation, by decreasing the weight of occluded blocks according to variation of block relationship coefficient.

In our method, the color histograms of current block and its neighbor blocks are extracted. Where, neighbor blocks are chosen as the upside, downside, left side and right side blocks of the current block. After color histograms are extracted, the Bhattacharyya distance [14] is used to metric the similarity of color histogram between current block and its neighbors.

$$
B C_{u}=\text { Bhattacharyya }\left(\text { Hist }_{0}, \text { Hist }_{u}\right)
$$

In formula (4), Hist H $_{0}$ represents the color histogram of current block with standing for the color histogram of its upside block. In the same way, we can get $B C_{u}, B C_{d}, B C_{l}, B C_{r}$ which represent the Bhattacharyya distances of color histogram between current block and its upside, downside, left side and right side block respectively. In particle filter algorithm, lots of particles are seeded in next frame for search- 
ing the most matched object region. Each particle stands for a candidate region, and each candidate region is divided into same size blocks as same as current region. The Bhattacharyya distance of color histogram between candidate block and its neighbor blocks are calculated as $B C_{u}^{\prime}, B C_{d}^{\prime}, B C_{l}^{\prime}$ ,$B C_{r}^{\prime}$. We can see from the picture below.

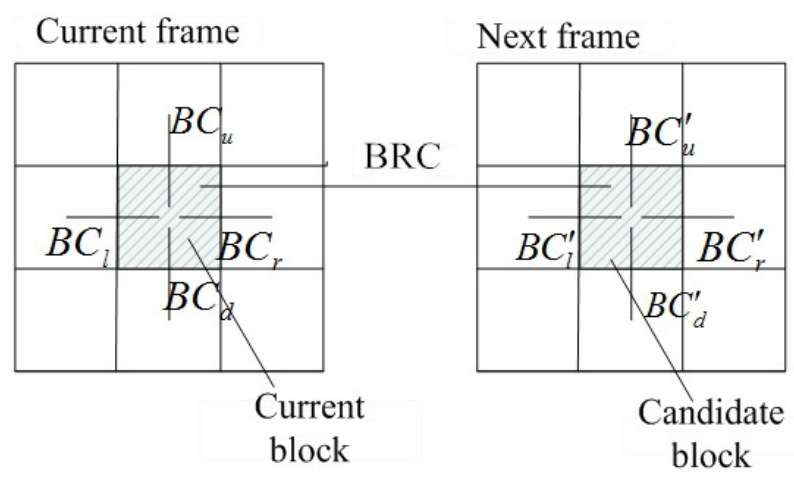

Figure 1: Illustration of BRC and BC of the current block.

Block relationship coefficient is defined as the variation of Bhattacharyya color histograms distance between blocks at the same position in current region and candidate region. Formula is shown as below.

$$
\begin{aligned}
& B R C_{u}=\left|1-\frac{B C_{u}-B C_{u}^{\prime}}{B C_{u}}\right| \quad B R C_{d}=\left|1-\frac{B C_{d}-B C_{d}^{\prime}}{B C_{d}}\right| \\
& B R C_{u}=\left|1-\frac{B C_{u}-B C_{u}^{\prime}}{B C_{u}}\right| \quad B R C_{d}=\left|1-\frac{B C_{d}-B C_{d}^{\prime}}{B C_{d}}\right|
\end{aligned}
$$

From the formula (5 )and (6) we can see BRC can represent the relationship between current block and its neighbors. The smaller BRC is, the robuster the block is. That means the interference to this block is small, so the weight of this block is increased in voting process. The use of BRC can help tracking algorithm to achieve a good performance in condition of occlusion and abrupt posture variation.

\section{2 proposed method's strategy}

The strategy of proposed method is shown as below: Step1. The rectangle of targeted object region is defined by people in the first frame. According to the rectangle size, current region is divided into several blocks with same size. Then the color histogram is extracted as the feature of each block. For each block, calculate the Bhattacharyya distance of color histogram between it and its neighbor blocks as $B C_{u}, B C_{d}, B C_{l}, B C_{r}$.

Step2. Use particle filter to seed particles in next frame. Each particle represents a candidate region which has the same size with current region. Do the same operation to candidate region as to current region to get the color histogram of each block. And calculate the Bhattacharyya distance of color histogram between it and its neighbor blocks as $B C_{u}^{\prime}, B C_{d}^{\prime}, B C_{l}^{\prime}, B C_{r}^{\prime}$

Step3. Use $B C$ and $B C^{\prime}$ to compute BRC of the two blocks which local in the same position in current and candidate region respectively. At the meantime, compute the color histogram Bhattacharyya distance of the two blocks, multiplied by $\mathrm{BRC}$ as the weight in voting process.

Step4. Switch to step2 until all the particles are seeded. Then all the blocks are voted to identify the most matched region. The region whose weight is higher than other regions is the most matched region. The rectangle of this region is the tracking result of this method.

\section{EXPERIMENTAL RESULTS}

The proposed method is completed in MATLAB and runs on a $2.5 \mathrm{GHz}$ i5-2450M Core PC with $8 \mathrm{G}$ memory. Standard video sequences named "caviar" and "occlusion" are chosen as the testing data in experiment. In order to show the advantage of the proposed method based on BRC, the traditional particle filter tracking algorithm and blocking particle filter tracking algorithm [11] are used for comparison. For convenience, they are represented by "traditional" and "blocking" in the figures of experimental results. The three tracking algorithms' tracking results in video "caviar" are shown as Fig.1.

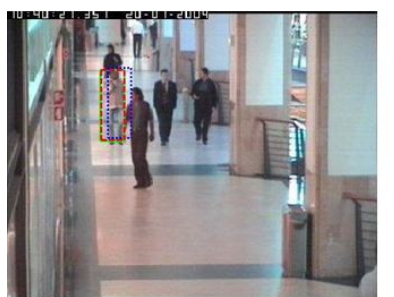

(a)

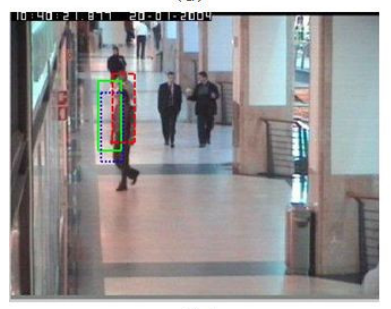

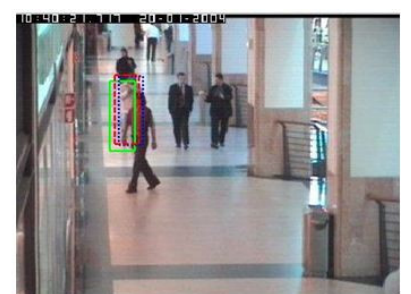

(b)

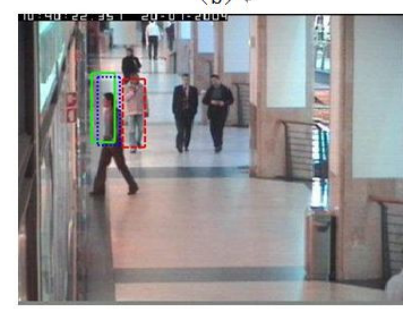

(d)

-
Figure 2: Tracking performance comparison of different algorithms in the "caviar" video sequence.

From Fig.2a and Fig.2b, we can see all the three algorithms can track the object accurately when there is no occlusion occurs to the object. However, if occlusion happens to the targeted object, tracking accuracy of traditional and blocking particle algorithms would be influenced greatly. From Fig.2c and Fig.2d we can see, the proposed method based on BRC can track the object even half of the object is hidden by occlusion. The proposed method has the better robustness than other two algorithms.

Fig. 3a shows that all the algorithms can track the woman's face accurately in the beginning. From Fig. 3b, Fig. 3c and Fig. 3d we can see that, while the woman's face is covered by the book, the accuracy of traditional and blocking particle filter algorithms is reduced. As the block relationship coefficient reduces the influence of occlusion, the proposed 


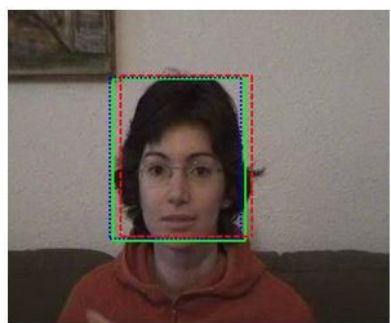

(a)

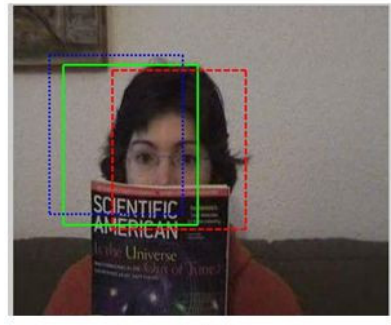

(c)

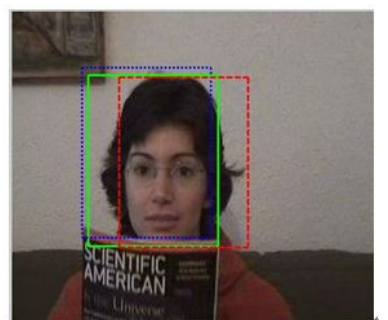

(b)

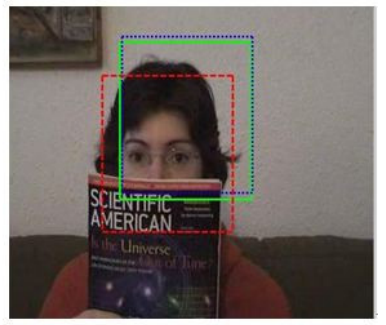

(d)

- proposed
Figure 3: Tracking performance comparison of different algorithms in the "occlusion" video sequence.

method has good tacking performance in condition of occlusion.

\section{CONCLUSIONS}

In this paper, we propose the block relationship coefficient which is used to increase the robustness of tracking algorithm based on particle filter. The weight of each block is identified according to the BRC while blocks are voted on the most matched region in next frame. Experimental results show that the proposed method can provide good performance in object tracking process.

\section{REFERENCES}

[1] Grigorios Tsagkatakis and Andreas Savakis. Online distance metric learning for object tracking. 21(12):1810-1821, 2011.

[2] Wei Zhong, M Yang, et al. Robust object tracking via sparse collaborative appearance model. 2014.

[3] Om Prakash, Manish Khare, Rajneesh Kumar Srivastava, and Ashish Khare. Tracking of deformable object in complex video using steerable pyramid wavelet transform. In Computational Vision and Robotics, pages 1-6. Springer, 2015.

[4] Weiming Hu, Tieniu Tan, Liang Wang, and Steve Maybank. A survey on visual surveillance of object motion and behaviors. 34(3):334-352, 2004.

[5] Dorin Comaniciu, Visvanathan Ramesh, and Peter Meer. Kernel-based object tracking. 25(5):564-577, 2003.

[6] Jia Zhang, Huiqi Li, Qing Nie, and Li Cheng. A retinal vessel boundary tracking method based on bayesian theory and multi-scale line detection. Computerized Medical Imaging and Graphics, 38(6):517-525, 2014.

[7] Jaegeol Yim, Seunghwan Jeong, Kiyoung Gwon, and Jaehun Joo. Improvement of kalman filters for wlan based indoor tracking. Expert Systems with Applications, 37(1):426-433, 2010.

[8] Mónica F Bugallo, Shanshan Xu, and Petar M Djurić. Performance comparison of ekf and particle filtering methods for maneuvering targets. Digital Signal Processing, 17(4):774-786, 2007.

[9] Zhaowen Wang, Xiaokang Yang, Yi Xu, and Songyu $\mathrm{Yu}$. Camshift guided particle filter for visual tracking. Pattern Recognition Letters, 30(4):407-413, 2009.

[10] Changjiang Yang, Ramani Duraiswami, and Larry Davis. Efficient mean-shift tracking via a new similarity measure. In Computer Vision and Pattern Recognition, 2005. CVPR 2005. IEEE Computer Society Conference on, volume 1, pages 176-183. IEEE, 2005.

[11] Amit Adam, Ehud Rivlin, and Ilan Shimshoni. Robust fragments-based tracking using the integral histogram. In Computer vision and pattern recognition, 2006 IEEE Computer Society Conference on, volume 1, pages 798-805. IEEE, 2006.

[12] Peihua Li, Tianwen Zhang, and Arthur EC Pece. Visual contour tracking based on particle filters. Image and Vision Computing, 21(1):111-123, 2003.

[13] Li Guanbin and Wu Hefeng. Weighted fragments-based meanshift tracking using color-texture histogram [j]. Journal of Computer-Aided Design \& Computer Graphics, 12, 2011.

[14] Meifeng Shi, Zhongshi He, Xin Liu, and Meiyan Huang. A distance weight object tracking method based on combining mean shift and gm $(1,1)$. JDCTA: International Journal of Digital Content Technology and Its Applications, 6(1):318-325, 2012. 